Screening kann Leben retten

\title{
Ab 65 steigt das Risiko für ein Bauchaortenaneurysma
}

\begin{abstract}
Die plötzliche Ruptur eines Bauchaortenaneurysmas endet in 90\% der Fälle tödlich. Eine rechtzeitige Intervention könnte die Patienten davor schützen. Für Risikopatienten empfiehlt sich daher ein Screening per Ultraschall.
\end{abstract}

- Bei $80 \%$ der Patienten bleibt ein abdominelles Aortenaneurysma (AAA) asymptomatisch. Verursacht die Gefäßerweiterung Beschwerden, dann sind diese meist sehr unspezifisch wie z.B. Bauch- oder Rückenschmerzen. Aus diesem Grund werden die meisten AAA nur zufällig entdeckt. Kommt es vorher zur Ruptur, sind die Folgen dramatisch. Nur jeder fünfte Patient mit einer AAA-Ruptur schafft es überhaupt ins Krankenhaus, 90\% der Betroffenen sterben infolge der Blutung.

\section{Männer ab 65 besonders gefährdet}

Aneurysmen entwickeln sich infolge einer chronischen Entzündung der Gefäßwand. Bei jedem fünften Patienten liegt eine genetische Disposition zugrunde. Besonders gefährdet sind Männer ab 65 Jahren. Bei 5,5\% der über 65jährigen Männer und 1,3\% der über 65-jährigen Frauen wurde in Screeninguntersuchungen ein AAA mit einem Durchmesser von mindestens $3 \mathrm{~cm}$ gefunden. Hochgerechnet auf die Einwohnerzahl müsste ein AAA also allein bei etwa 100000 Männern und 35000 Frauen über 65 Jahren in Deutschland vorliegen. Auch bei Rauchern, Hypertonikern und KHK-Pati-

\section{Tabelle 1}

\section{Risikofaktoren für AAA}

- Zunehmendes Lebensalter

- Positive Familienanamnese

- Männliches Geschlecht

- Aktueller oder früherer

Nikotinabusus

- Koronare Herzkrankheit

- Arterielle Hypertonie enten besteht ein erhöhtes Risiko für ein AAA (Tab. 1).

\section{Operation bei großen Aneurysmen}

$\mathrm{Ab}$ einem Durchmesser des AAA von $5 \mathrm{~cm}$ steigt das Rupturrisiko steil an. Deshalb ist bei Männern ab 5-5,5 cm und bei Frauen ab 4,5-5 cm eine Operation indiziert. Behandelt wird das Aneurysma entweder in einer offenen Operation, in der die abdominelle Aorta durch eine Rohr- oder Bifurkationsprothese ersetzt wird, oder durch die Implantation einer Stentprothese über die Leistenarterien.

\section{Screening halbiert die Mortalität}

Eine frühzeitige Diagnose ist der einzige Weg, die Gefahr, die von einem AAA ausgeht, zu verringern. Erkennbar sind AAA in Ultraschalluntersuchungen. In einigen Ländern, z. B. in den USA, in Großbritannien und in Schweden, wurden bereits Screeningprogramme eingeführt. In Deutschland gibt es bisher kein generelles Screening, aber die Ultraschalluntersuchung kann als IGeL angeboten werden. Auf Initiative der Deutschen Gesellschaft für Gefäßchirurgie und Gefäßmedizin (DGG) findet am 8. Mai der Tag des BauchaortenaneurysmaScreenings statt, an dem in vielen Kliniken kostenlose Screeninguntersuchungen möglich sind.

Den Nutzen eines AAA-Screenings belegen vier Studien mit insgesamt über 135000 Personen. Drei Studien schlossen nur Männer über 65 Jahren ein. Patienten mit großen AAA wurden an einen Gefäßchirurgen überwiesen, Patienten mit kleinen AAA regelmäßig

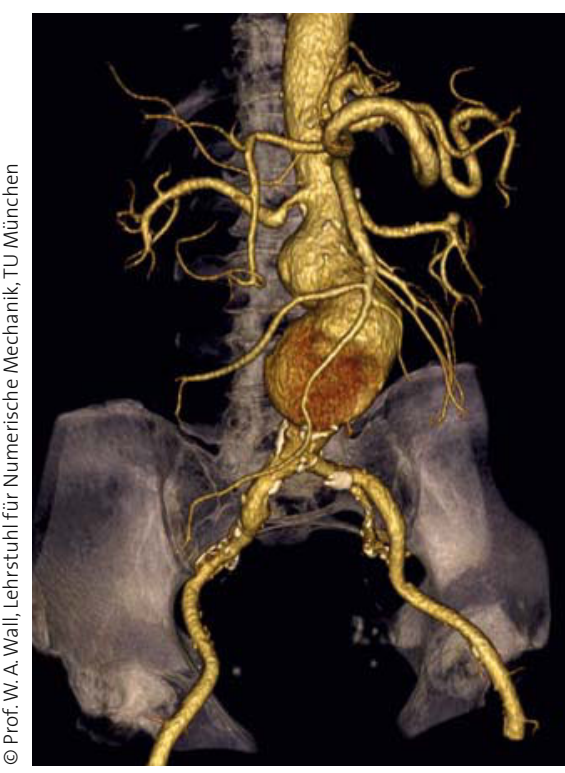

Bauchaortenaneurysma im 3-D-CT.

nachuntersucht. Durch dieses Vorgehen konnte die AAA-bedingte Mortalität um 44\% reduziert werden.

\section{Screening für wen?}

Die DGG empfiehlt eine einmalige Ultraschalluntersuchung der abdominalen Aorta bei

- Männern ab dem 65. Lebensjahr, insbesondere bei ehemaligem oder aktuellem Nikotinabusus,

- Männern und Frauen aller Altersstufen mit positiver Familienanamnese,

- Frauen ab dem 65. Lebensjahr mit (vorbestehendem) Nikotinabusus, kardiovaskulärer Vorgeschichte und/ oder positiver Familienanamnese.

Dr. JUdith Neumaier .

- Informationen der DGG; Böckler D et al. Gefäßchirurgie 2009;14:350-361

\section{Mai: Tag des Screenings}

\section{Kliniken bieten kosten-} lose Untersuchungen an

Am 8. Mai gibt es in zahlreichen Kliniken die Möglichkeit für ein kostenloses Ultraschallscreening, mit dem Ziel, bisher unerkannte Bauchaortenaneurysmen zu entdecken. Initiator des Screening- und Informationstags ist die DGG. Eine Liste aller teilnehmenden Kliniken gibt es unter www.gefaesschirurgie.de. 